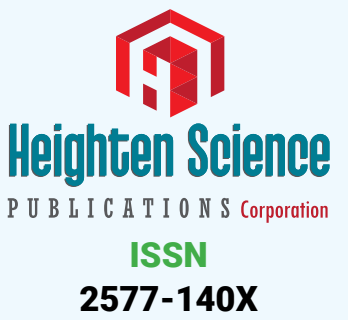

*Address for Correspondence: Ayse Gul Kocak Altintas, MD, Associated Professor, University of Health Sciences, Ankara Ulucanlar Eye Education and Research Hospital, Ankara, Turkey, Tel: 90-312-3126261; Fax: 90-312-3124827; Email: aysegulkaltintas@hotmail.com

Submitted: 15 March 2018

Approved: 22 March 2018

Published: 23 March 2018

Copyright: @2 2018 Altintas AGK, et al.,. This is an open access article distributed under the Creative Commons Attribution License, which permits unrestricted use, distribution, and reproduction in any medium, provided the original work is properly cited

Keywords: Aflibercept; Bevacizumab; Pigment epithelial detachment; Ranibizumab
Review Article

\section{Treatment of Retinal Pigment Epithelial Detachment}

\author{
Ayse Gul Kocak Altintas ${ }^{1 *}$ and Cagri Ilhan ${ }^{2}$ \\ ${ }^{1}$ Associated Professor, University of Health Sciences, Ankara Ulucanlar Eye Education and \\ Research Hospital, Ankara, Turkey \\ ${ }^{2}$ Hatay State Hospital, Hatay, Turkey
}

\section{Abstract}

Anatomical separation of the retinal pigment epithelium from the Bruch membrane is defined as retinal pigment epithelial detachment (PED) andit is classified as drusenoid, serous, and vascularized. Vascularized PED is mostly associated with choroidal neovascularmembrane due to age-related macular degeneration and the risk of vision loss is high in this situation. Studies show that all of baseline values including BCVA, PED height, subretinal fluid, central macular thickness, PED volume, vertical dimension, presence of coincident macular pathology, reflectivity and morphology on optical coherence tomography have prognostic importance. Current treatment protocols mainly based on intravitreal injection of anti-vascular endothelial growth factor (VEGF). Even the bevacizumab was the first anti-VEGF that was used for treatment in PED, there are several reports show the insufficiency of bevacizumab. In treatment-naïve eyes, both of ranibizumab and aflibercepthave similar effect in vascularized PED. In treatment-resistant eyes, high dose bevacizumab or switching therapy of anti-VEGF procedures can be effective when considering of all cases, aflibercept seems more effective than other options. We aimed in this manuscript, to give a general information about different characteristics of PEDs and to investigate the treatment strategies in the light of current literature.

\section{Introduction}

Anatomical separation of the retinal pigment epithelium (RPE) from the Bruch membrane is defined as retinal pigment epithelial detachment (PED). Several degenerative and idiopathic ocular conditions and many systemic etiologies including renal, inflammatory, infectious, neoplastic, and iatrogenic reasons cause PED [1]. Agerelated macular degeneration (AMD) is the most common reason of PED and it can be associated with underlying choroidal neovascularization (CNV) [1]. The presence of PED associated with AMD is classified as drusenoid, serous, vascularized, or mixed according to different clinical and imaging findings. We aimed in this manuscript, to give a general information about different characteristics of PEDs and to investigate the treatment strategies in the light of current literature.

\section{Classification of pigment epithelial detachments}

Drusenoid PED is associated with confluent soft drusen and it comprises a high risk for dry AMD.Confluent soft drusen areas and sub-RPE space enlarge because stressed RPE is unable to remove fluid and debris [2].Fundoscopic appearing of drusenoid PED cannot be distinguished from large soft or confluent drusen which have scalloped border and a slightly irregular surface. Smooth contour of the detached hyperreflective RPE band and hyporeflective area beneath the RPE are occurred in optical coherence tomography (OCT) and it is generally not associated with intraretinal or subretinal fluid. Figure 1 shows an OCT image section and macular thickness map of drusenoid PED (Figure 1). DrusenoidPED typically isofluorescent or hyperautofluorescent in fundus autofluorescence (FAF) [3]. On fundus fluorescein angiography (FFA), it usually shows 

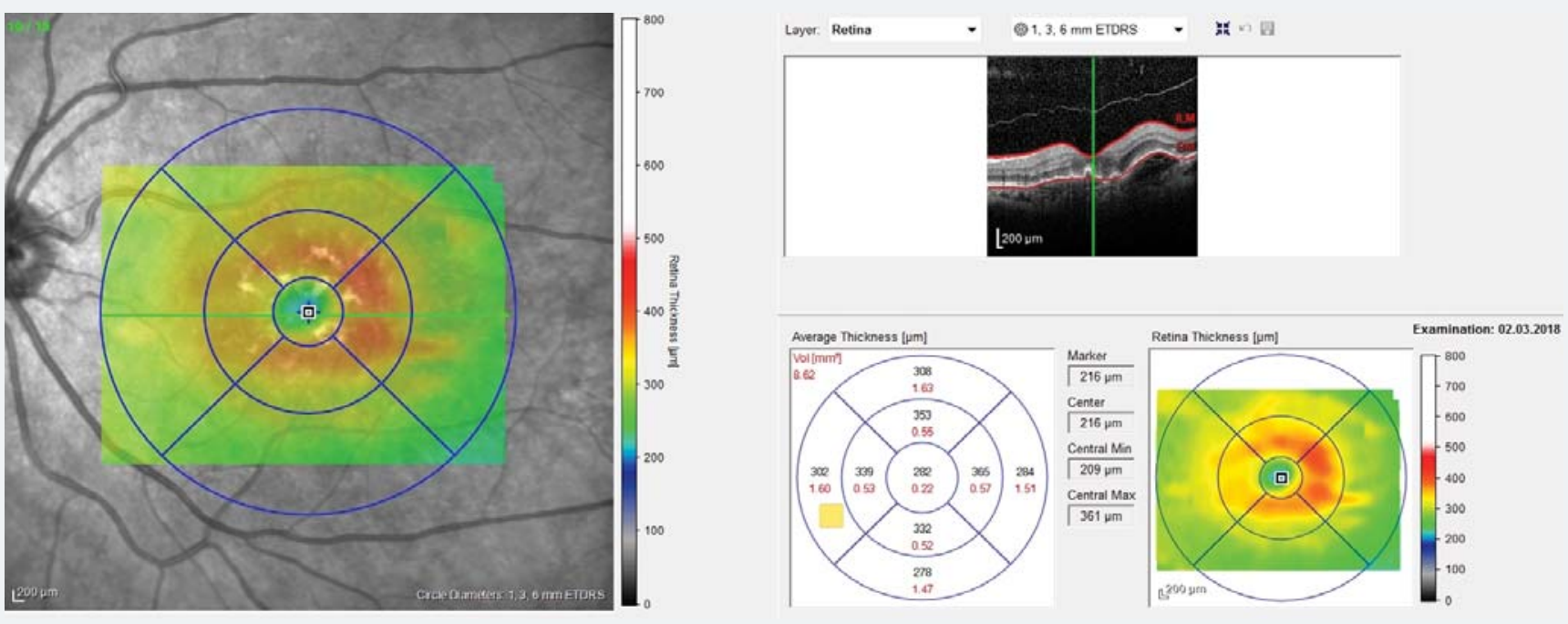

Figure 1: Macular thickness map of drusenoid pigment epithelial detachment

faint hyperfluorescence correspond to the window defect and focal hypofluorescence correspond to the blockage effect. With indocyanine green angiography (ICGA), drusenoid PED appears as a homogeneous hypoflueorescent lesion [4].

According to Casswell et al., study, anatomical and visual outcomes of drusenoid PEDs are better than other types of PEDs [5]. The chance of spontaneous resolution of drusenoid PED with a legacy of RPE atrophy is high [6].

Serous PED is caused by fluid collection between RPE and Bruch membrane and it occurs as sharply demarcated elevations of the RPE. The reasons of fluid collection are increased choriocapillaris leakage and decreased RPE pump function. AMD and central serous chorioretinopathy are two of the most common diseases associated with serous PED $[7,8]$.The classical fundus finding of serous PED is distinct circular ovoid elevation. The typical well-demarcated, dome-shaped elevation that is characteristic finding of serous PED are best seen in OCT. In FAF, serous PED is seen hyperautofluorescence in hypoautofluorescence border [9]. It is determined with early hyperfluorescence and progressive well-demarcated pooling in the area of PED, by FFA. Serous PED is seen hypofluorescent in ICGA. The possibility of spontaneous resolution with pigment epithelial atrophy is higher in serous PED developed secondary to central serous chorioretinopathy than AMD.

Vascularized PED is mostly associated with type 1 or occult CNV but any other subfoveal pathologies can be associated with it. In clinical examination, vascularized PED is appeared as irregular solid elevation of the RPE with or without intraretinal exudates. In OCT evaluation, solid, fluidly and hyperreflective material along the back surface of RPE, is found [10]. Figure 2 shows intraretinal and subretinalhyporeflective spaces in OCT section in case of vascularized PED (Figure 2). FAF signals are increased in vascularized PED due to its hyperrefletive solid components [9]. According to FFA, there are two types of occult CNV including fibrovascular PED and vascularized PED which characterized with late-phase leakage [11,12]. In ICGA, it is shown late hyperfluorescence with the presence of occult CNV [12]. According to ICGA, hyperfluorescent area is descripted in two forms such as a hot spot which is smaller than 1 disc diameter and plaque which is larger than 1 disc diameter.

Vascularized PEDs have increased risk of developing to disciform scarin natural course [13]. RPE tear is occurred about $10 \%$ this rate can be accelerated after antivascular endothelial growth factor (VEGF) therapy $[5,14]$. 

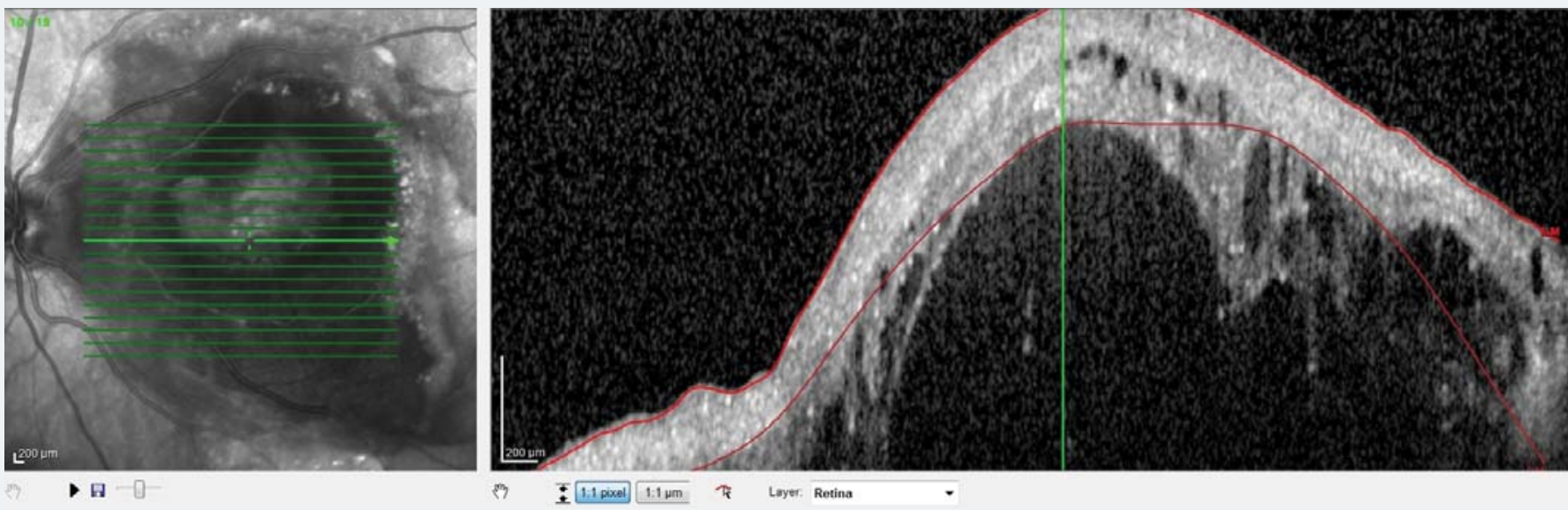

Figure 2: Optical coherence tomography section in vascularized pigment epithelial detachment.

The natural course of vascularized PED is progressive visual loss which can be occurred suddenly due to hemorrhage or RPE tear. The risk of vision loss is high in vascularized PED [1]. Generally, FFA is inadequate to differentiate of serous and vascularized PED because both of them have the characteristics of early hyperfluorescence and late staining. ICGA is a benefit method in differential diagnose. Early hyperfluorescence and very slow staining are occurred in serous PED while rapidly hyperfluorescence and evident staining are occurred in vascularized PED [15]. After the correct differential diagnosis, treatment is necessary due to possible poor prognosis of vascularized PED.

Treatment is not necessary for some cases in these situations including absence of symptoms and good visual acuity. This strategy is rational when considering the potential complications of treatment methods that can be very devastating such as macular scar due to retinal photocoagulation, or endophthalmitis due to intravitreal injection etc. Baba et al., reported that avascular PEDs had a better visual acuity and intravitreal antiVEGF is not quietly useful in these cases [16]. Because, fluid accumulation between RPE and choriocapillaris caused by hydrophobic barrier of Bruch's membrane, not CNV and anti-VEGF treatment is controversial.The anatomical and functional prognosis of these cases are better than others with neovascularization [16].

\section{Current treatment of vascularized pigment epithelial detachment}

Vascularized PED is a common complication of neovascular AMD. In past, total ablation with laser photocoagulation was used to performed for treatment of vascularized PED but even it prevents progression of membrane functional outcomes of this treatment were not satisfactory due to laser scars [17]. The applicability of photodynamic treatment or other modalities are decreased day by day in intravitreal pharmacotherapy era. Current treatment protocols include intravitreal injection of anti-VEGF for the treatment of PED.

The efficacy of anti-VEGF injection was first described in bevacizumab. Yüksel et al., reported that intravitreal bevacizumab therapy preserved vision after 1-year followup and decreased PED height [18]. On the other hand, according to Ach et al., study, bevacizumab treatment cannot significantly decrease in PED height in vascularized PED over a period of 9 months [19]. Lee et al., investigated that the effects of high dose intravitreal bevacizumab (5mg) on treatment of persisted PED in neovascular AMD [20]. They found that after the repeating (mean 3.39 times) high dose intravitreal bevacizumab injection, best corrected visual acuities (BCVA) are increased, central foveal thickness and PED height were statistically significantly decreased and they did not observe any serious adverse effects. They stated that increasing the dose of bevacizumab may be a possible treatment option in case of treatment-resistant PED [20]. 
In treatment-naïve eyes pro-re-nata protocol of ranibizumab therapy is found to be beneficial. Chevreaud et al., reported mean central macular thickness decreased from $572.1 \mu \mathrm{m}$ to $396.6 \mu \mathrm{m}$, while the mean height of PED decreased from $458.2 \mu \mathrm{m}$ to 306.8 $\mu \mathrm{m}$ and mean BCVA increased from 0.46 to $0.39 \operatorname{logMAR} 12$ months after the treatment [21]. According to their study vascularized PED in neovascular AMD responded well to ranibizumabin terms of functional and anatomical outcomes.

Aflibercept is a VEGF-trap molecule that have more powerful binding affinity than anti-VEGFagents. According to VIEW1 and VIEW 2 studies, after the 3-monthly loading regimen,aflibercept can be injected less frequently than other anti-VEGF agents in neovascular AMD [22]. On the other hand, there are several studies that compare the effects of ranibizumab and aflibercet in vascularized PED and nearly all of them showed that both them are effective in treatment-naïve eyes. Vaze et al., reported that both of these anti-VEGF therapies provide significant functional and anatomical improvements in treatment-naïve eyes ofneovascular AMD with vascularized PED [23]. They also observed in 6-months follow-up, the mean number of injections and visits were similar in 2 drug groups[23]. On the contrary, Balaskas et al., found aflibercept is more effective than ranibizumab according to anatomic and functional results of $3^{\text {rd }}$ and $12^{\text {th }}$ months [24].

Switching treatment from ranibizumab to aflibercept seems a good choice in unresponsive PEDs. In treatment-resistant AMD,switching to aflibercept improved both visual and anatomical outcomes. Kumar et al., found a significant reduction in central retinal thickness in eyes with neovascular AMD, 97\% of them had PED, 3-months after switching toaflibercept [25]. Additionally, Chan et al., reported significant functional and anatomic improvements 6-months after switching to aflibercept,in their series of 189 eyes with AMD. They stated that the response of switching was better in eyes with PED than the eyes without PED [26].

De Massougnes et al., found that the visual improvement is associated with the baseline characteristics including lower BCVA, presence ofsubfoveal fluid, and female gender. PED height decrement is positively associated with higher baseline PED height, the higher the baseline PED height the more reduction was observed [27]. According to Balascas et al., study, all of baseline values including BCVA, subretinal fluid, central macular thickness, PED volume, PED's vertical dimension have prognostic importance [24]. In contrary, there are many studies argued that baseline characteristics of PED have not any influence on the functional outcomes [23].

According to recent studies the reflectivity of PED on OCT was found as one of the most important prognostic factors that indicate the response of anti-VEGF therapy in which hyporeflective PEDs are more treatable than hyperreflective PEDs [1,10,28-30]. The hyporeflective PED's response to anti-VEGF therapy is more satisfactory in terms of reduction of subretinal fluid volume, RPE volume, and BCVA. It is also found that hyperreflective elevations are more associated with occult CNV [1].

Lam et al., separated PEDsinto 2 groups according to their morphology by OCT as eyeswith wrinkled vascularized PED and without wrinkled vascularized PED. They stated that wrinkled vascularized PED is associated with better BCVA and it needs less prevalent intravitreal injection [15]. According to their hypothesis,wrinkled surfaced PED may occur due to contracture of the material within PED when height of PED is already diminished and that cause better response to anti-VEGFtreatment [15]. Possibly RPE tears are also less common in these eyes.

The response of aflibercept treatment is also determined by presence of coincident macular diseases such as CNV and its subtypes.Choroidal vessel proliferation locates beneath the RPE causes type $1 \mathrm{CNV}$ while type $3 \mathrm{CNV}$ also known as retinal angiomatous 
proliferation originates from deep retinal capillary vessels. The baseline anatomical and clinical characteristics and also functional outcomes in follow-up period are differed in vascularized PED with type 1 than type 3 CNV. Chen et al., reported PED with type $3 \mathrm{CNV}$ is better response to aflibercept than PED with type $1 \mathrm{CNV}$ and it needs less frequent aflibercept injection [31].

Kim et al., reported the development of several adverse eventsafter the aflibercept treatment in eyes with refractory to previous continuous anti-VEGF injection including, epiretinal membrane, vitreous and subretinal hemorrhage. These complications were mainly occurredin eyes with PED associated subretinal fluid [32]. According to Traumer et al., study in the series of 132 case with neovascular AMD, most of the eyes has submacularhemorrhagewere associated with PED [33]. The presence of PED seems to an important predisposing factor for occurrence of submacular hemorrhage.

Vaze et al., reported the occurrence of RPE tear in bothranibizumab and aflibercept treatment groups [23]. Actually, RPE tears can occur in natural course of vascularized PED associated with neovascular AMD in range of 10-12\%. All types of anti-VEGF injections increase the risk of RPE tear in around 12-25\% [34]. PED size and height are also important risk factors for RPE [35]. Doguizi et al., determined $580 \mu \mathrm{m}$ as a cutoff value for RPE tear after anti-VEGF injection [34]. Rapid contraction of the proliferated membranes under the RPE is a probably mechanism and these tears commonly occur in post-injection $1^{\text {st }}$ or $2^{\text {nd }}$ months [34].

In conclusion, intravitreal aflibercept treatment is seemed as the most effective treatment option in neovascular AMD with vascularized PED. Nevertheless, difference of ranibizumab and aflibercet is very little in treatment-naïve eyes. In treatmentresistant eyes, even high dose bevacizumab injection was applied but switching is seemed as more current protocol. The prognosticimportance of baseline characteristics is controversy but reflectivity and morphology of lesion by OCT or present coincidences effects on functional and anatomical outcomes.

\section{References}

1. Mrejen S, Sarraf D, Mukkamala SK, Freund KB. Multimodal imaging of pigment epithelial detachment: a guide to evaluation. Retina. 2013; 33: 1735-1762. Ref.: https://goo.gl/3PMfj4

2. Sarks JP, Sarks SH, Killingsworth MC. Evolution of soft drusen in age-related macular degeneration. Eye (Lond). 1994; 8: 269-283. Ref.: https://goo.gl/KyUxNF

3. Schmitz-Valckenberg S, Fleckenstein M, Scholl HP, Holz FG. Fundus autofluorescence and progression of age-related macular degeneration. Surv Ophthalmol. 2009; 54: 96-117. Ref.: https://goo.gl/KXVte5

4. Arnold JJ, Quaranta M, Soubrane G, Sarks SH, Coscas G. Indocyanine green angiography of drusen. Am J Ophthalmol. 1997; 124: 344-356. Ref.: https://goo.gl/HUxH5g

5. Casswell AG, Kohen D, Bird AC. Retinal pigment epithelial detachments in the elderly: classification and outcome. Br J Ophthalmol. 1985; 69: 397-403. Ref.: https://goo.gl/KhUZFh

6. Hartnett ME, Weiter JJ, Garsd A, Jalkh AE. Classification of retinal pigment epithelial detachments associated with drusen. Graefes Arch Clin Exp Ophthalmol. 1992; 230: 11-19. Ref.: https://goo.gl/Wtr106

7. Lommatzsch A, Heimes B, Gutfleisch M, Spital G, Zeimer M, et al.,. Serous pigment epithelial detachment in age-related macular degeneration: comparison of different treatments. Eye (Lond). 2009; 23: 2163-2168. Ref.: https://goo.gl/26chZh

8. Laatikainen L, Hoffren M. Long-term follow-up study of non- senile detachment of the retinal pigment epithelium. Eur J Ophthalmol. 1991; 1: 79-84. Ref.: https://goo.gl/SkBViT

9. Karadimas P, Bouzas EA. Fundus auto fluorescence imaging in serous and drusenoid pigment epithelial detachments associated with age-related macular degeneration. Am J Ophthalmol. 2005: 140: 1163-1165. Ref.: https://goo.gl/8FpL2E

10. Spaide RF. Enhanced depth imaging optical coherence tomography of retinal pigment epithelial detachment in age-related macular degeneration. Am J Ophthalmol. 2009; 147: 644-652. Ref.: https://goo.gl/D28pK5 
11. Subfoveal neovascular lesions age-related macular degeneration. Guidelines for evaluation and treatment in the macular photocoagulation study Group. Arch Ophthalmol. 1991; 109: 1242-1257. Ref.: https://goo.gl/eQi13W

12. Yannuzzi LA, Slakter JS, Sorenson JA, Guyer DR, OrlockDA. Digital indocyanine green videoangiography and choroidal neovascularization. Retina.1992; 12: 191-223. Ref.: https://goo.gl/YUCeuh

13. Gass JD. Drusen and disciform macular detachment and degeneration. Arch Ophthalmol.1973; 90: 206-217. Ref.: https://goo.gl/pTuYBs

14. Chang LK, Flaxel CJ, Lauer AK, Sarraf D. RPE tears after pegaptanib treatment in age-related macular degeneration. Retina. 2007; 27: 857-863. Ref.: https://goo.gl/jd1oRd

15. Lam D, Semoun O, Blanco-Garavito R, Jung C, Nguyen DT, et al.,. Wrinkled vascularized retinal pigment epithelium detachment prognosis after intravitreal anti-VEGF therapy. Retina. 2017. Ref.: https://goo.gl/fccRT5

16. Baba T, Kitahashi M, Kubota-Taniai M, Oshitari T, Yamamoto S. Two-year course of subfoveal pigment epithelial detachment in eyes with age-related macular degeneration and visual acuity better than 20/40. Ophthalmologica. 2012; 228: 102-109. Ref.: https://goo.gl/pTohtv

17. Brancato R, Introini U, Bolognesi G, Pacelli G, Trabucchi G, et al.,. ICGA-guided laser photocoagulation of occult choroidal neovascularization in age-related macular degeneration. Indocyanine green angiography. Retina. 2000; 20: 134-142. Ref.: https://goo.gl/66A4Zw

18. Yüksel $H$, Türkcü FM, Sahin $A$, Sahin $M$, Cinar $Y$, et al...One year results of anti-VEGF treatment in pigment epithelial detachment secondary to macular degeneration. Arq Bras Oftalmol. 2013; 76: 209-211. Ref.: https://goo.gl/cZewgP

19. Ach T, Hoeh AE, Ruppenstein M, Kretz FT, Dithmar S. Intravitreal bevacizumab in vascular pigment epithelium detachment as a result of subfoveal occult choroidal neovascularization in age-related macular degeneration. Retina. 2010; 30: 1420-1425. Ref.: https://goo.gl/ZDXuuq

20. Lee DK, Kim SH, You YS, Kwon OW. High Dose Intravitreal Bevacizumab for Refractory Pigment Epithelial Detachment in Age-related Macular Degeneration. Korean J Ophthalmol. 2016; 30: 265271. Ref.: https://goo.gl/rDrx7e

21. Chevreaud O, Oubraham $H$, Cohen SY, Jung $C$, Blanco-Garavito $R$, et al... Ranibizumab for vascularized pigment epithelial detachment: 1-year anatomic and functional results. Graefes Arch Clin Exp Ophthalmol. 2017; 255: 743-751. Ref.: https://goo.gl/4fJCBL

22. Heier JS, Brown DM, Chong V, Korobelnik JF, Kaiser PK, et al.,. Intravitreal aflibercept (VEGF trap-eye) in wet age-related macular degeneration. Ophthalmology. 2012; 119: 2537-2548. Ref.: https://goo.gl/c8ibsz

23. Vaze A, Nguyen V, Daien V, Arnold JJ, Young SH, et al.,. Ranibizumab and aflibercept for the treatment of pigment epithelial detachment in neovascular age-related macular degeneration: Data from an Observational Study. Retina. 2017. Ref.: https://goo.gl/CbU1hP

24. Balaskas K, Karampelas M, Horani M, Hotu O, Keane $\mathrm{P}$, et al.,. Quantitative analysis of pigment epithelial detachment response to different anti-vascular endothelial growth factor agents in wet age-related macular degeneration. Retina. 2017; 37: 1297-1304. Ref.: https://goo.gl/gGCLwD

25. Kumar N, Marsiglia M, Mrejen S, Fung AT, Slakter J, et al.,. Visual and anatomical outcomes of intravitreal aflibercept in eyes with persistent sub-foveal fluid despite previous treatments with ranibizumab in patients with neovascula rage-related macular degeneration. Retina. 2013; 33: 16051612. Ref.: https://goo.gl/1YiS5U

26. Chan CK, Jain A, Sadda S, Varshney N. Optical coherence tomographic and visual results at six months after transitioning to aflibercept for patients on prior ranibizumab or bevacizumab treatment for exudative age-relatedmacular degeneration (an AmericanOphthalmologicalSocietythesis). Trans Am Ophthalmol Soc. 2014; 112: 160-198. Ref.: https://goo.gl/he65fj

27. deMassougnes S, Dirani A, Mantel I. Good visual outcome at 1 year in neovascular age-related macular degeneration with pigment epithelium detachment: Factors Influencing the Treatment Response. Retina. 2018. 38: 717-724. Ref.: https://goo.gl/yCFMw7

28. Broadhead GK, Hong T, Zhu M, Li H, Schlub TE. Response of pigment epithelial detachments to intravitreal aflibercept among patients with treatment-resistant neovascular age-related macular degeneration. Retina. 2015; 35: 975-981. Ref.: https://goo.gl/ejVYW2

29. He L, Silva RA, Moshfeghi DM, Blumenkranz MS, Leng T. Aflibercept for the treatment of retinal pigment epithelial detachments. Retina. 2016; 36: 492-498. Ref.: https://goo.gl/TsWakB 
30. Zinkernagel MS, Wolf S, Ebneter A. Fluctuations in pigment epithelial detachment and retinal fluid using a bimonthly treatment regimen with aflibercept for neovascular age-related macular degeneration. Ophthalmologica. 2016; 235: 42-48. Ref.: https://goo.gl/gXKB5D

31. Chen X, Al-Sheikh M, Chan CK, Hariri AH, Abraham P, et al.,. Type 1 versus type 3 neovascularization in pigment epithelial detachments associated with age-related macular degeneration after antivascular endothelial growth factor therapy: A Prospective Study. Retina. 2016; 36: 50-64. Ref.: https://goo.gl/xreoH3

32. Kim K, Kim ES, Kim Y, Yang JH, Yu SY, et al.,. Outcome of intravitreal aflibercept for refractory pigment epithelial detachment with or without subretinal fluid and secondary to age-related macular degeneration. Retina. 2017. Ref.: https://goo.gl/ZaLU51

33. Treumer F, Wienand S, Purtskhvanidze K, Roider J, Hillenkamp J. The role of pigment epithelial detachment in AMD withs submacular hemorrhage treated with vitrectomy and subretinal coapplication of rtPAand anti-VEGF. Graefes Arch Clin Exp Ophthalmol. 2017; 255: 1115-1123. Ref.: https://goo.gl/EWmZRB

34. Doguizi S, Ozdek S. Pigment epithelial tears associated with anti-VEGF therapy: incidence, longterm visual outcome, and relationship with pigment epithelial detachment in age-related macular degeneration. Retina. 2014; 34: 1156-1162. Ref.: https://goo.gl/bBdxkX

35. Chiang A, Chang LK, Yu F, Sarraf D. Predictors of anti-VEGF-associated retinal pigment epithelial tear using FA and OCT analysis. Retina. 2008; 28: 1265-1269. Ref.: https://goo.gl/BwhsNe 